\title{
SCIDiC
}

\author{
International Journal of Dentistry and Oral Science (IJDOS) \\ ISSN: 2377-8075
}

\section{Comparative Evaluation Of Antibacterial Efficacy Of Two Chewing Sticks Against Enterococcus Faecalis - An In Vitro Study \\ Research Article}

TamizhPaavai. Tha ${ }^{1}$, Jayashri Prabakar ${ }^{2 *}$, S. Sneha ${ }^{3}$

${ }^{1}$ Saveetha Dental College, Saveetha Institute of Medical and Technical Sciences Saveetha University, Chennai, India.

${ }^{2}$ Senior Lecturer, Department of Public Health Dentistry, Saveetha Dental College, Saveetha Institute of Medical and Technical Sciences Saveetha University, Chennai, India.

${ }^{3}$ Senior Lecturer, Department of Oral Medicine and Radiology, Saveetha Dental College, Saveetha Institute of Medical and Technical Sciences, Saveetha University, Chennai, India.

\section{Abstract}

Background: The use of chewing sticks for maintain oral hygiene is a common ancient practice still followed in parts of South East Asia. Many naturally occurring plants are used for this purpose. However, their actions against specific organisms are not fully established. In this study, the antibacterial efficiency of two chewing sticks commonly used in South India namely neem and banyan against Enterococcus faecalis, the most common oral pathogen responsible for root canal failures.

Aim: To compare and evaluate the antibacterial efficacy of chewing sticks against Enterococcus faecalis.

Materials and Methods: The neem and banyan twigs were obtained from the local markets and were cut into approximately $15 \mathrm{~cm}$ length. They were further sun dried for two days before grounding them into coarse powders. The extracts obtained from the chewing sticks are diluted into aqueous solutions of varied concentrations of 1:5, 1:10 and 1:15 and subjected to Minimum inhibitory concentration evaluation. The zones of inhibition were measured and assessed for its antibacterial activity against Enterococcus faecalis.

Result: It was observed that the antimicrobial efficacy of both the extracts increased with their increase in concentration. But the action of neem was more potent than banyan.

Conclusion: This study conducted suggests that the naturally occurring components of the chewing sticks can be a good alternative for the currently available root canal irrigants that might not guarantee the success of treatment due to their inability to combat E.faecalis.

Keywords: Antimicrobial; Banyan; Extract; E. faecalis; Neem.

\section{Introduction}

Oral health plays a pivotal role in maintaining a good general health of a person. The main disease to affect the oral cavity is the dental caries. With more awareness among the general population, the importance to maintain good oral hygiene has also increased among the people. In the olden days, it was observed that in many parts of the world the people used chewing sticks to clean their teeth, which were primarily made from the branches of various trees present in that ethnic area. Many surveys conducted in many countries suggested that the prevalence of dental caries in the rural areas where chewing sticks were still used for oral hygiene was lower or comparable to the oral hygiene of their counterparts who live in the urban areas [1]. These findings are attributed to the varied food practices in these areas or the natural products present in these chewing sticks. There have been many studies that tested the efficiency of chewing sticks to be used as an alternative to toothbrushes for daily oral hygiene practices.

Chewing sticks are most commonly used in parts of the Middle East, India and Africa. It is observed to be more suitable for cleansing the oral cavity, cost efficient, ease of use and is also observed to have many medicinal properties. It is a product that requires no expertise to be produced [2]. There are about 173 dif-

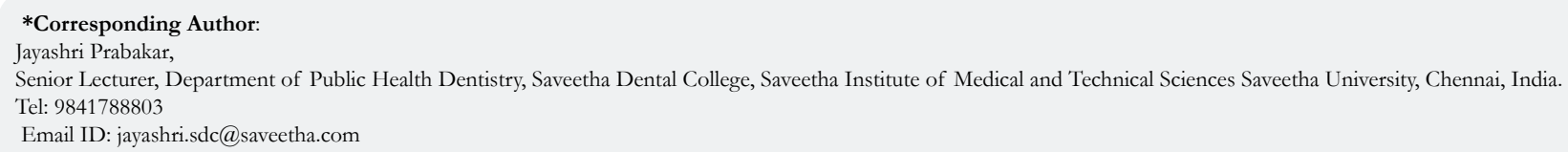

Citation: TamizhPaavai. Tha, Jayashri Prabakar, S. Sneha. Comparative Evaluation Of Antibacterial Efficacy Of Two Chewing Sticks Against Enterococcus Faecalis - An In Vitro Study. Int J Dentistry Oral Sci. 2021;08(03):1878-1881. doi: http:/ /dx.doi.org/10.19070/2377-8075-21000373

Copyright: Jayashri Prabakar ${ }^{\circ}$ 2021. This is an open-access article distributed under the terms of the Creative Commons Attribution License, which permits unrestricted use, distribution and reproduction in any medium, provided the original author and source are credited. 
ferent types of trees that are used as chewing sticks. They belong to various species that include Acacia Fabaceae, Terminalia, Combretaceae, Lasianthera, Icacinaceae, Gouania, and Rhamnaceae [3, 4]. The antimicrobial action of these chewing sticks mainly to naturally protect the plants from parasites that attacks them. It is believed that these agents will help to combat the cariogenic and periodontopathic microorganisms in the oral cavity. The chewing sticks are attributed to have many properties such as antibacterial, astringent, detergent, anti inflammatory, abrasive and plaque inhibiting. It is also observed that they enhance salivary production, improve bone and tooth development, fight caries, reduce stains naturally and prevents plaque [5].

Banyan (Ficus benghalensis) is a plant that grows on another plant (epiphyte) when their seeds germinate in cracks and crevices on a host tree. The disposable toothbrushes are made from the aerial roots of the banyan. These roots have astringent properties that help to whiten teeth and also strengthen teeth and the periodontium. It is believed that the chewing sticks are good mechanical plaque remover which helps remove yellow stains in teeth [6].

Neem, Nimtree, Margosa tree or Indian Liliac known as Azadirachta indica belongs to the family Meliaceae. It is primarily found in the Indian subcontinent that includes Nepal, Pakistan, Bangladesh and Sri Lanka. It is one of the most versatile plants that are widely used in medicinal preparations. It is recognized as "a tree for solving global problems" by the United States National Academy of Science [6]. To use the twigs of the neem tree as chewing sticks one end is chewed to form soft bristles to clean the oral cavity by brushing. Once used as a toothbrush it is converted into a tongue cleaner by chewing. It is observed in many surveys that approximately $80 \%$ of the rural population in India still uses neem-chewing sticks to clean their teeth and their urban counterparts prefer neem toothpastes. It is found to be effective in reducing plaque and gingival inflammation [7]. Neem possessesastringent, antiseptic properties that help treat halitosis and fight cariogenic microorganisms in the oral cavity.

The method of evaluation used in this study is measurement of the zone of inhibition for each extract at varied concentrations. The minimum inhibitory concentration is defined as the lowest concentration of an antimicrobial that inhibits the visible growth of a microorganism after overnight intubation whereas minimum bactericidal concentration refers to the lowest concentration of an antimicrobial that inhibits the growth of an organism after a subculture on to an antibiotic-free media. This is the most common method for initial evaluation of any product prior to in vivo studies to be conducted in animal and human trials. This helps to estimate its efficacy and biocompatibility of the test substance under ideal conditions before they are exposed to in vivo studies.

\section{Materials and Methods}

\section{Preparation of chewing sticks}

The twigs of the locally available neem and banyan were obtained from the local markets that were identified by their color and scent. These twigs were cut into approximately $15 \mathrm{~cm}$ length (Figures 1 and 2). They were further sun dried for two days before grounding them into coarse powders. The aqueous extracts for each stick was prepared by mixing $5 \mathrm{gm}, 10 \mathrm{gm}$ and $15 \mathrm{gm}$ of chewing stick powder with $100 \mathrm{ml}$ of deionized distilled water. The mixture obtained was shaken well manually and allowed to soak for 48 hours at $4^{\circ} \mathrm{C}$. These solutions were later filtered to obtain $5 \%, 10 \%$ and $15 \%$ concentration of each type of chewing stick.

\section{Anti-Microbial Assay}

The strain of Enterococcus faecalis was taken. The media used was blood infused agar. This media is mainly composed of a protein source primarily tryptones from the pancreatic digest of casein, papaic digest of soy meal, sodium chloride, agar and 5\% sheep blood along with distilled water. They are adjusted to a $\mathrm{pH}$ of 7.3. The organism was inoculated in the selected medium using swab method. Sterile discs were incorporated with equal amount of different concentrations of the prepared chewing stick formulations using a micropipette. These discs were later placed equidistant from each other and were further incubated for 24 hours.

\section{Measurement of Zone of Inhibition}

The test plated were held below a desk lamp, and the zones were measured using a ruler placed against the back of the petri dish. The diameter of the zones of inhibited growth were measured to the nearest whole milllimeter.

\section{Results}

Table 1 depicts the action of neem in varied dilutions against Enterococcus faecalis. The zone of inhibition is found to more as the concentration of neem increases from $5 \%, 10 \%$ and $15 \%$ that measure $2 \mathrm{~mm}, 7 \mathrm{~mm}$ and $10 \mathrm{~mm}$ respectively. This suggests that the inhibitory action of neem against the test organism increases with its increase in concentration. It is observed from Table 2, that the initial dilution of Banyan with a concentrationof 1:5 does not exhibit any inhibitory effect against the testing organism but the zone of inhibition is found to more as the concentration of banyan increases from $10 \%$ to $15 \%$ that measure $1 \mathrm{~mm}$ and $5 \mathrm{~mm}$ respectively. This suggests that the inhibitory action of banyan against the test organism increases with its increase in concentration.

Tabel 1. Zone of inhibition of neem against E.faecalis organism.

\begin{tabular}{|c|c|}
\hline \multicolumn{2}{|c|}{ NEEM } \\
\hline Dilution & Zone of Inhibition (in $\mathbf{~ m m}$ ) \\
\hline $1: 05$ & 2 \\
\hline $1: 10$ & 7 \\
\hline $1: 15$ & 10 \\
\hline
\end{tabular}


Table 2. Zone of inhibition of banyan against E.faecalis organism.

\begin{tabular}{|c|c|}
\hline \multicolumn{2}{|c|}{ BANYAN } \\
\hline Dilution & Zone of Inhibition (in $\mathbf{~ m m}$ ) \\
\hline $1: 05$ & 0 \\
\hline $1: 10$ & 1 \\
\hline $1: 15$ & 5 \\
\hline
\end{tabular}

Figure 1. Chewing sticks of neem.

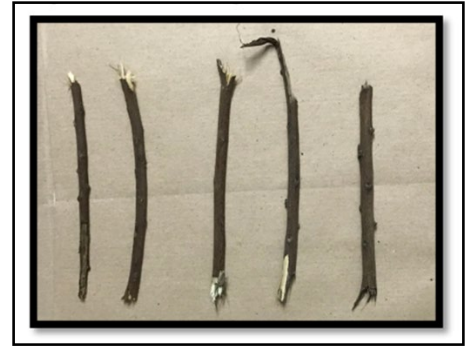

Figure 2. Chewing sticks of banyan.

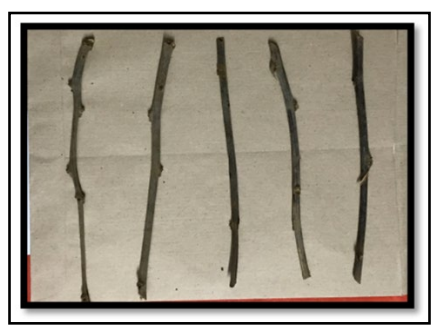

\section{Discussion}

The role of many micro organism have been explained to be the primary cause for the formation of pulp and periapical diseases. This has been demonstrated by many animal models and human studies coducted by various authors [8]. The major root canal infections are primarily polymicrobial that mostly comprise of obligatory anaerobic bacteria. The most commonly isolated bacteria from the root canal include anaerobic Gram-negative rods, Lactobacillus species, Gram-positive anaerobic cooci, faculatative rods and Streptococcus species.

Eventhough the obligate anaerobes are easily removed by irrigation of the root canal, the facultative bacteria such as Staphylococcus and Enterococcus that once enter a canal are seen to survive chemicomechanical instrumentation and the root canal [9]. In the recent years, particular emphasis has been made on the Enterococcus species in case of endodontic literature as they have been isolated frequently from the root canals of failed root canal treatments [9].

The use of herbal products as an alternative to the current treatment modalities has been the topic of research in the recent times. Many traditional plants have been studied for their biological and antimicrobial properties, which is relevant to the current situation of antibiotic overuse and misuse. The most commonly tested plants in the field of dentistry include phytomedicines, which are observed to have anti inflammatory, antibiotic, analgesic and sedative properties [10].

The neem plant also known as Azadirachta indica is one of the most versatile medicinal plant that possess a wide spectrum of biological activity ad had been used in traditional Indian medicine for more than 2000 years. This plant is mainly investigated due to its antimicrobial potential against several microorganisms and also they're anti adherence activity by altering bacterial adhesion and thus preventing the colonization of an organism. A study conducted by Bithelho et al and Singhal et al also suggest that Azadirachta indica is highly effective in the treatment of periodontal disease which present their biocompatibility to human periodontal ligament fibroblasts $[11,12]$. Thus the use of neem as an irrigant is beneficial since it is a biocompatible antioxidant and most likely would not cause severe injuries to patient that may occur with sodium hypochlorite which is the most commonly used root canal irrigants in recent times.

The results of the current study suggest that both the neem and banyan extracts have antimicrobial efficacy against E. faecalis and their activity increased with higher concentrations. The extracts of neem showed higher antimicrobial action against the test organism E.faecalis than banyan, which is similar to the other studies done previously. A study conducted by Prasanth et al that compared the antimicrobial efficacy of neem and mango extracts presented with more effectiveness of neem when compared to mango against carious microorganisms [2]. Khalid et al also presented with similar results when comparing neem and Arak extract efficacy at different concentrations against microorganisms [13]. The study conducted by Wolinsky and his collegues subjected saliva-contained hydroxyapatite to neem stick extract prior to bacterial exposure which presented with reduced bacterial adhesion thus suggesting that neem extracts have an increased potential to inhibit plaque formation [14]. The antimicrobial action of neem may be attributed to its many features. These include presence of fluoride of about $1.0 \mu \mathrm{g} / \mathrm{g}$ and silica. The fluoride acts as an anti cariogenic action while the silica helps prevent plaque accumulation. The neem extracts also contain alkaloids, which help in dental health. The various alkaloids present in neem include resins, gum, chloride, fluoride, silica, margosine, sulfur, tannins, 
oils, saponins, flavonoids, sterols, calcium, phenolic compounds, carotenoids, steroids, triterpenoids, valvenoids, ketones and also tetranortriterpenoid azadirachtin that help in their antimicrobial action [15].

The banyan also known as Ficus benghalensis has multiple effects against various organisms. A study conducted by Gaythri et al using the bark of banyan showed their antibacterial efficacy against S.aureus and P.aeruginosa and Klebsiella pnuemoniae [16]. The aqueous aerial root extracts of banyan in varied concentrations of 25,50 and $75 \mathrm{mg} / \mathrm{ml}$ were observed to have anti bacterial action against many organism and the highest inhibitory action was found against S.aureus [17]. Another study conducted in the year 1994 by Mousa et al suggested that the fruit extracts of the banyan had antibacterial action but do not possess antifungal properties [18]. Murti and Kumar in the year 2011 reported from their study that different concentrations of the ethanolic extracts of the roots showed moderated action against S.aureus, P.aeruginosa and K.pnumonia [19]. The various types of extract of the banyan were checked for their antibacterial efficacy at high and low concentrations against K.pneumoniae, P.aeruginosa, E.coli, E.faecalis, P.vulgaris, B.subtilis and Micrococcus luteus by Koona and Rao in the year 2012. This study when done using hex leaves extracts revealed that at lower concentrations they presented with immunity against K.pneumoniae, P.aeruginosa and Micrococcus luteus but exhibited intermediate action against K.pneumoniae in high concentrations [20]. But it was observed that they produced no action against E.coli, E.faecalis, P.vulgaris, and B.subtilis. However when the same organisms were subjected to chloroform extracts of banyan, it was observed that they were effective against K.pnuemoniae and M.luteus; presented with intermediate activity against P.aeruginosa in any concentration given. The organisms E.coli, P.vulgaris and Enterococcus faecalis had intermediate resistance when subjected to high concentrations and no resistance when exposed to low concentration. It was observed that B.subtilis presented with no inhibition zones irrespective of the concentration they were subjected to. When exposed to methanol extracts, all organisms showed resistant activity for both high and low concentrations [20]. The methanol, ethyl acetate and acetone extracts of the bark of banyan tree wereexposed to different microorganisms by Manimozhi et al. showed good antibacterial efficacy against P.aeruginosa, E.coli, P.vulgaris, B.subtilis and S.aureus [21].

One of the recent studies conducted in the year 2012 by Elangovan et al [1]. showed that when the extracts of banyan were exposed to E.faecalis showed no inhibitory action irrespective of its concentration. This result is contradictory to the results obtained in the present study wherein the extracts showed minimal inhibitory action at high concentrations.

From this study it is observed that anti microbial efficacy of neem is better than banyan extracts and can be considered as a potential root canal irrigant in place of the current synthetic alternatives available in the market.

\section{Conclusion}

This study primarily tests two naturally occurring product extracts against one of the most common oral microorganism in case of root canal infections are considered. The study also enumerates the other beneficialeffects of these extracts against other systemic organisms that might enter the host organism through the oral route. Their multiple phytochemical components such as tannins, flavonoids and flavonols must be evaluated to a greater extent that can help to efficiently use these natural products that produce less side effects than the current medicaments and irrigants available which can cause potential damage to the host tissues that compromises the success of the treatment.

\section{References}

[1]. Elangovan A, Muranga J, Joseph E. Comparative evaluation of the antimicrobial efficacy of four chewing sticks commonly used in South India: an in vitro study. Indian J Dent Res. 2012 Nov-Dec;23(6):840.Pubmed PMID: 23649081.

[2]. Prashant GM, Chandu GN, Murulikrishna KS, Shafiulla MD. The effect of mango and neem extract on four organisms causing dental caries: Streptococcus mutans, Streptococcus salivavius, Streptococcus mitis, and Streptococcus sanguis: an in vitro study. Indian J Dent Res. 2007 Oct-Dec;18(4):148-51. Pubmed PMID: 17938488.

[3]. Dogan AU, Dogan M, Chan DC, Wurster DE. Bassanite from Salvadora persica: a new evaporitic biomineral. Carbonates Evaporites. 2005 Mar;20(1):2-7.

[4]. Njoroge GN, Kaibui IM, Njenga PK, Odhiambo PO. Utilisation of priority traditional medicinal plants and local people's knowledge on their conservation status in arid lands of Kenya (Mwingi District). J Ethnobiol Ethnomed. 2010 Aug 16;6:22.Pubmed PMID: 20712897.

[5]. Ahmad M, Imran H, Yaqeen Z, Rehman Z, Rahman A, Fatima N, et al. Pharmacological Profile of Salvador persica. Res Inst Pharm Sci Pak J Pharm Sci 2011; 24(3): 323-30

[6]. Sagar S. Role of natural toothbrushes in containing oral microbial flora-A review. Asian J Pharm Clin Res. 2015;8(4):29-33.

[7]. Bhambal A, Kothari S, Saxena S, Jain M. Comparative effect of neemstick and toothbrush on plaque removal and gingival health-A clinical trial. J. Adv. Oral Res. 2011 Oct;3(3):51-6.

[8]. Mohammadi Z. Sodium hypochlorite in endodontics: an update review. Int Dent J. 2008 Dec;58(6):329-41.Pubmed PMID: 19145794.

[9]. Zehnder M. Root canal irrigants. J. Endod. 2006 May 1;32(5):389-98.

[10]. Francisco Carlos Groppo et al, Phytotherapy Research; 2008; 22(8): 993998.

[11]. Anurag S, Anuraag G, Chandrawati G. Comparison of antimicrobial efficacy of conventional irrigants and herbal products alone and with calcium hydroxide against entercoccus faecalis. Guident. 2012;5(2):77-9.

[12]. Botelho MA, dos Santos RA, Martins JG, Carvalho CO, Paz MC, Azenha C, et al. Efficacy of a mouthrinse based on leaves of the neem tree (Azadirachta indica) in the treatment of patients with chronic gingivitis: A double-blind, randomized, controlled trial. J Med Plants Res. 2008 Nov 30;2(11):341-6.

[13]. Khalid A. Antimicrobial effects of Azadirachta indica (Neem) and Salvadora persica (Arak) chewing sticks. Indian J Dent Res 1999; 10:23-6.

[14]. Wolinsky LE, Mania S, Nachnani S, Ling S. The inhibiting effect of aqueous Azadirachta indica (Neem) extract upon bacterial properties influencing in vitro plaque formation. J Dent Res. 1996 Feb;75(2):816-22.Pubmed PMID: 8655780 .

[15]. Kraus W. Biologically active ingredients. In: Schmutterer H, editor. The Neem tree: Source of unique natural products for integrated pest management, medicine, industry and other purposes. Weinheim: VCH. 1995. 12(5); 35-88.

[16]. Gayathri M, Kannabiran K. Antimicrobial activity of Hemidesmus indicus, Ficus bengalensis and Pterocarpus marsupium roxb. Indian J Pharm Sci. 2009 Sep;71(5):578-81.Pubmed PMID: 20502584. https://pubmed.ncbi. nlm.nih.gov/20502584/

[17]. Singh RK, Geeta W. Antimicrobial potential of Ficus bengalensis aerial roots. Int. J. Pharm. Biol. Sci. 2010;1(3):1-9.

[18]. Mousa O, Vuorela P, Kiviranta J, Wahab SA, Hiltunen R, Vuorela H. Bioactivity of certain Egyptian Ficus species. J Ethnopharmacol. 1994 Jan;41(12):71-6.Pubmed PMID: 8170162.

[19]. Murti K, Kumar U. Antimicrobial activity of Ficus benghalensis and Ficus racemosa roots L. Am. J. microbiol. 2011;2(1):21-4.

[20]. Koona SJ, Rao BS. In vitro evaluation of antibacterial activity of crude extracts of Ficus benghalensis Linn., the banyan tree leaves. Indian J. Natural Products Resour. 2012; 3:281-284.

[21]. Manimozhi DM, Sankaranarayanan S, Kumar GS. Effect of different extracts of stem bark of Ficus sp. on multidrug resistant pathogenic bacteria. Int. J. Pharm. Sci. Res. 2012 Jul 1;3(7):2122-2129. 